\title{
Atmospheric parameters and chemical composition of the ultra-cool roAp star HD $213637^{\star}$
}

\author{
O. Kochukhov ${ }^{\star \star}$ \\ Uppsala Astronomical Observatory, Box 515, SE-751 20 Uppsala, Sweden \\ Received 14 January 2003 / Accepted 31 March 2003

\begin{abstract}
A high-resolution spectrum obtained with the UVES instrument at the ESO VLT is used to determine atmospheric parameters and chemical composition of the roAp star HD 213637. Resolved Zeeman split lines are discovered in the spectrum of this star, indicating a mean field modulus of $5.5 \mathrm{kG}$. Effective temperature, $T_{\text {eff }}=6400 \mathrm{~K}$ determined from the profiles of the hydrogen $\mathrm{H} \alpha$ and $\mathrm{H} \beta$ lines, makes HD 213637 one of the coolest (ro)Ap stars known, suggesting that the class of magnetic chemically peculiar stars extends to lower effective temperatures than previously thought. Furthermore, the low temperature of HD 213637 poses serious challenge to some excitation mechanisms suggested as an explanation of the roAp phenomenon. This peculiar star is also unique in its evolutionary stage: it is either located near the very end of its main sequence life or has already ascended the giant branch. Abundance analysis reveals that HD 213637 is iron-deficient and has abundance pattern typical of the cool pulsating Ap stars. An inconsistency between abundances derived from the weak and strong spectral lines and the lines of different ions provide a clear evidence for prominent vertical abundance stratification of many chemical species, notably $\mathrm{Na}, \mathrm{Mg}, \mathrm{Ca}, \mathrm{Fe}, \mathrm{Pr}, \mathrm{Nd}, \mathrm{Tb}$, and $\mathrm{Er}$.
\end{abstract}

Key words. stars: abundances - stars: chemically peculiar - stars: individual: HD 213637 - stars: magnetic fields stars: oscillations

\section{Introduction}

Rapidly oscillating Ap (roAp) stars form a subgroup of cool magnetic chemically peculiar stars that spans an effective temperature range from about $7000 \mathrm{~K}$ to $8500 \mathrm{~K}$. The pulsations of roAp stars are non-radial, high overtone, acoustic $p$-modes with periods from 5 to $20 \mathrm{~min}$ and semi-amplitudes typically below 10 mmag in Johnson $B$ (Kurtz \& Martinez 2000). Detailed studies of the pulsations in roAp stars allow inferring the evolutionary status and internal properties of magnetic peculiar stars via the application of the asteroseismological analysis. Since the discovery of the roAp pulsations (Kurtz \& Wegner 1979), the mechanism which is responsible for the excitation of non-radial oscillations and distinguishes between pulsating and, otherwise very similar but apparently constant, cool Ap stars remains a mystery. Systematic determination of the atmospheric parameters and detailed abundance analysis of a large sample of roAp stars can provide important observational constraints for theoretical models aiming at the explanation of the roAp phenomenon. Such work has been carried out since the mid-90s and has yielded information on chemical composition and atmospheric properties of 9 roAp stars (Ryabchikova et al. 2000 and references therein). The present paper extends this analysis to the faint poorly studied roAp star HD 213637 ,

* Based on observations obtained at the European Southern Observatory, VLT.

$\star \star$ e-mail: oleg@astro.uu.se which appears to have extreme characteristics among the objects belonging to the group of pulsating Ap stars.

HD 213637 (MM Aqr, BD -20 6447 ) is a $V=9.6 \mathrm{mag}$ star, classified as EuSrCr by Houk \& Smith-Moore (1988). $p$-mode oscillations were discovered in HD 213637 by Martinez et al. (1998). They found evidence for the presence of two pulsation modes with amplitudes below $1 \mathrm{mmag}$ and tentatively assigned them to frequencies of 1411 and $1452 \mu \mathrm{Hz}$. The Strömgren colours of HD 213637 published by Martinez et al. indicate strong metallicity and unusually low effective temperature. According to the SIMBAD database, except for spectral classification (F2), no other spectroscopic abundance or model atmosphere studies of HD 213637 were published.

This paper is organized as follows: in Sect. 2 the spectroscopic observations of HD 213637 are described. Stellar atmospheric parameters are derived in Sect. 3, which is followed by the analysis of the rotation and magnetic field in Sect. 4. The results of the study of chemical composition are presented in Sect. 5 and the paper is concluded with the discussion in Sect. 6.

\section{Observations}

A single spectrum of HD 213637 was obtained on April 17, $2002(J D=2452381.905)$ with the UVES spectrograph at the Kueyen VLT unit telescope. The integration time was $600^{\mathrm{s}}$ and complete spectral coverage was obtained between 4774 

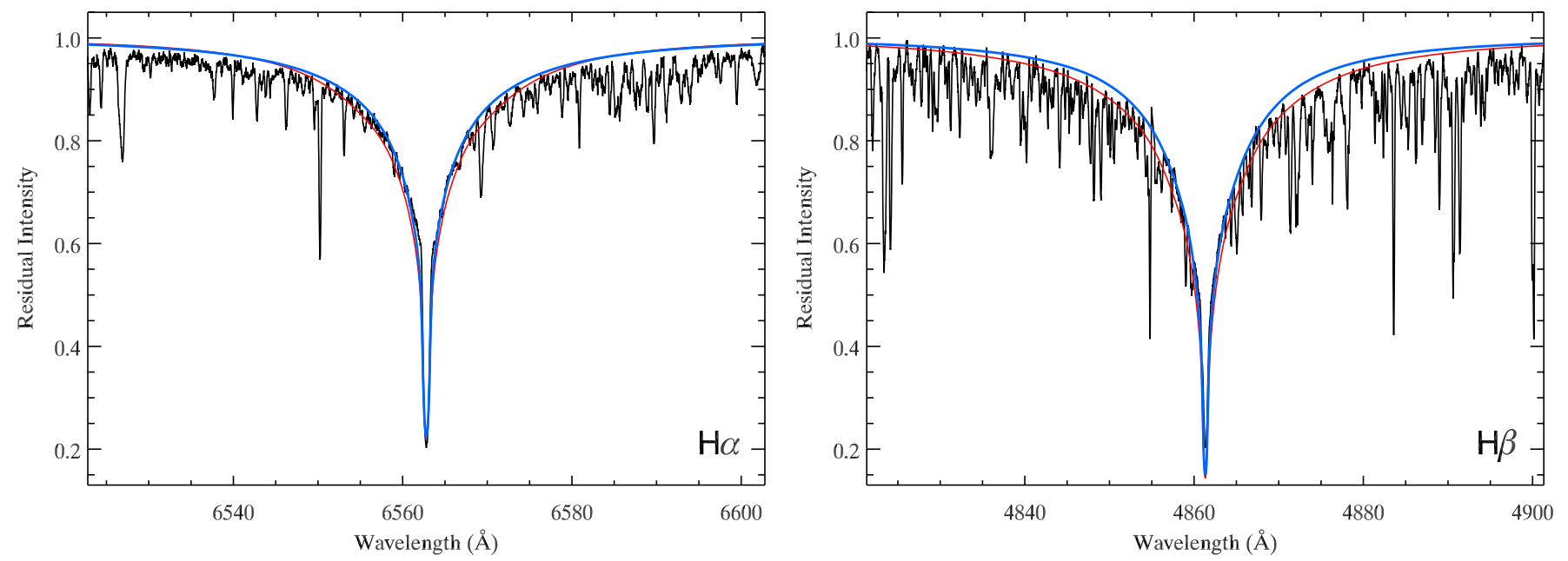

Fig. 1. Comparison between the observed profiles of the hydrogen $\mathrm{H} \alpha$ (left panel) and $\mathrm{H} \beta$ (right panel) lines and synthetic spectra computed using the model atmosphere with $T_{\text {eff }}=6400 \mathrm{~K}$ and $\log g=3.6$ (thick line). The thin curve illustrates the effect of increasing $T_{\text {eff }}$ by $200 \mathrm{~K}$.

and $6812 \AA$, except for a $60 \AA$ gap centred at $5790 \AA$. The slit of the spectrograph was set to $0.6^{\prime \prime}$, resulting in a resolving power of $\lambda / \Delta \lambda=67000$. Echelle spectrum was reduced with the standard UVES pipeline software and subsequently normalized to the continuum using iterative spline fitting in the regions containing metal lines. Low-order polynomials were used for normalization in the neighbourhood of the hydrogen $\mathrm{H} \alpha$ and $\mathrm{H} \beta$ lines. The signal-to-noise ratio of the 1-D extracted spectrum of HD 213637 is $250-300$. Few examples of this observational material, giving an impression of the quality of the data, are presented in Figs. 1, 3 and 4.

\section{Effective temperature and surface gravity}

The starting values of the model atmosphere parameters of HD 213637 were derived from the Strömgren indices reported by Martinez et al. (1998) $(V=9.611, b-y=0.298$, $m_{1}=0.206, c_{1}=0.411$ and $\left.\beta=2.670\right)$ and using software package TEMPLOGG (Rogers 1995). The Strömgren photometry yields $T_{\text {eff }}=6687 \mathrm{~K}, \log g=4.60$ and $[M / H]=$ +0.57 . Effective temperature was subsequently refined by fitting the hydrogen $\mathrm{H} \alpha$ and $\mathrm{H} \beta$ lines, which gave $T_{\text {eff }}=6400 \pm$ 100 K. Modelling of Balmer lines was based on Kurucz (1993) ATLAS9 model atmosphere code and modern formulation of the hydrogen line broadening theory summarized by Barklem et al. (2000) and implemented in the SYNTH code (Piskunov 1992). The fit to the Balmer line profiles is illustrated in Fig. 1.

Photometry and high-resolution spectra indicate enhanced line blanketing in HD 213637, but it turns out that light and iron-peak elements are not overabundant relative to the solar chemical composition and iron itself is probably underabundant by roughly a factor of 10 (see Sect. 5). Increased line blanketing comes primarily from the strong lines of rare-earth elements (REE). To account for this unusual chemical composition I calculated model atmospheres using Kurucz's opacity distribution functions corresponding to the metallicity of +0.5 , but at the same time adopting the solar abundance table in order to avoid an increase in electron number density, which should not occur in the atmosphere of HD 213637 since

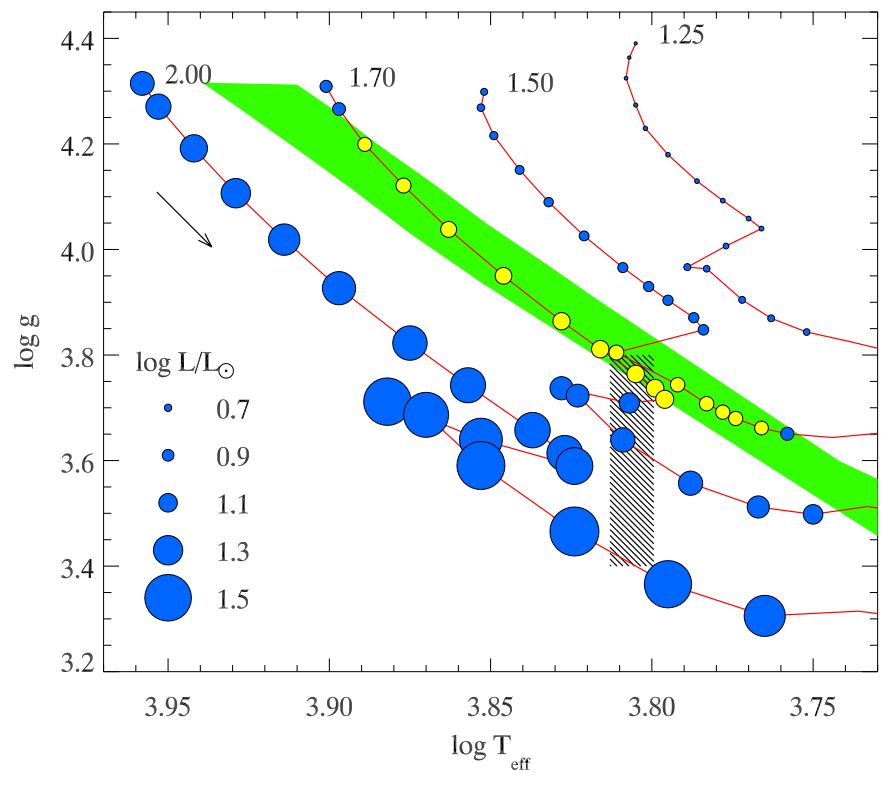

Fig. 2. The atmospheric parameters of HD 213637 determined in this study (dark shaded area) are compared with the theoretical stellar evolutionary calculations by Schaller et al. (1992). The tracks for models with initial masses of $1.25,1.5,1.70$, and $2.0 \mathcal{M}_{\odot}$ are plotted with the solid lines. Stars evolve from higher to lower surface gravities as indicated by the arrow. The circles correspond to individual models and circles' sizes are proportional to the stellar luminosity as explained in the legend. The open circles and light-shaded area highlight models which are consistent with the tentative asteroseismological luminosity of HD 213637.

primary donors of electrons are not overabundant. (Similar approach of accounting for an unusually high line blanketing was thoroughly tested by Cowley et al. (2000) in the spectroscopic analysis of Przybylski's star.) In addition, the opacity distribution function with microturbulent velocity of $4 \mathrm{~km} \mathrm{~s}^{-1}$ was employed in calculations with ATLAS9 in order to simulate the line strengthening produced by the strong field of HD 213637 via the magnetic intensification mechanism. 


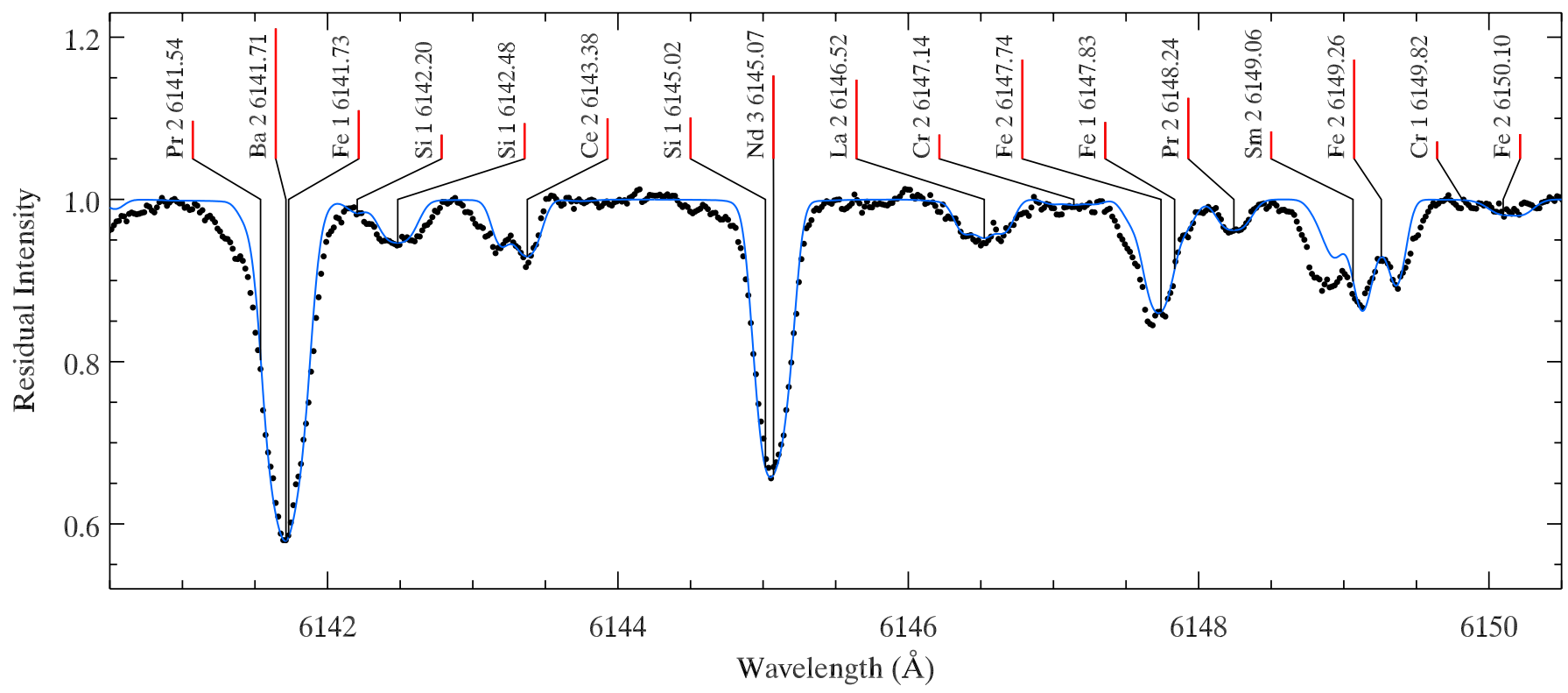

Fig. 3. Comparison between the observations in the 6140-6150 $\AA$ region (dots) and spectrum synthesis (solid line) for $B_{\mathrm{s}}=5.5 \mathrm{kG}$ and $v_{\mathrm{e}} \sin i=3.5 \mathrm{~km} \mathrm{~s}^{-1}$. Length of the lines underlining identification is proportional to the strength of corresponding spectral features. Note resolved Zeeman components of the Fe II $6149.26 \AA$.

The surface gravity indicated by the Strömgren photometry, $\log g=4.6$, is clearly too high for even a ZAMS star of 1.5-2.0 $\mathcal{M}_{\odot}$. Instead, ionization equilibrium of $\mathrm{Fe}$ I and $\mathrm{Fe}$ II was used to estimate $\log g$. This approach gave rather uncertain value of $\log g=3.6 \pm 0.2$. This large error comes from the fact that iron is clearly stratified in the atmosphere of HD 213637 (see Sect. 5.2) and it may not be justified to expect a priori the same abundance from lines of the two iron ions. The estimate of surface gravity could be improved by taking into account the vertical stratification of iron derived from, say, Fe I and Fe II lines.

The abundance analysis of HD 213637 presented in Sect. 5 was carried out with $T_{\text {eff }}=6400 \mathrm{~K}$ and $\log g=3.6$. I note that, despite high uncertainty of $\log g$, the value of effective temperature derived from the Balmer lines is fairly robust. In particular, the change of $\log g$ from 3.6 to 4.6 or/and variation of metallicity within 0.5 dex results in no more than $100 \mathrm{~K}$ change of $T_{\text {eff }}$ derived from $\mathrm{H} \alpha$.

The atmospheric parameters of HD 213637 are compared with the theoretical evolutionary tracks of Schaller et al. (1992) in Fig. 2. One can conclude that HD 213637 has a mass of $1.7 \pm 0.2 \mathcal{M}_{\odot}$ and is located very near the end of the core hydrogen burning phase or has already left the main sequence. An independent estimate of the luminosity of HD 213637 would be very helpful in refining its evolutionary state. Unfortunately accurate parallax of HD 213637 was not determined within the Hipparcos project. However, high-speed photometry by Martinez et al. (1998) hinted that several non-radial modes are excited in HD213637, opening interesting prospects for asteroseismology. The frequency separation of $\Delta v=41.46 \pm$ $0.13 \mu \mathrm{Hz}$ derived by Martinez et al. is subject to severe aliasing problems, but taking it at face value and combining with $T_{\text {eff }}$ estimated from the hydrogen lines, one can obtain asteroseismological luminosity $\mathcal{L}=10.8 \pm 2.1 \mathcal{L}_{\odot}$. This estimate is based on the relations given in Matthews et al. (1999), adopted stellar mass of $2.0 \pm 0.5 \mathcal{M}_{\odot}$ and an assumption that observed frequency spacing is due to consecutive overtones of modes with odd or even $\ell$. Tentative asteroseismological luminosity of HD 213637 can be reconciled with the theoretical evolutionary calculations and the effective temperature derived in this study if the stellar mass is $1.6 \pm 0.1 \mathcal{M}_{\odot}$ and surface gravity is $3.8 \pm 0.1$.

\section{Magnetic field and rotation}

The surface magnetic field is clearly strong in HD 213637. The splitting of the Fe II $6149.26 \AA$ indicates a mean field modulus of $B_{\mathrm{s}}=5.5 \pm 0.1 \mathrm{kG}$ (Fig. 3). This value is confirmed by the detailed spectrum synthesis of other Fe I and Fe II spectral lines with large Landé factors. Thanks to slow rotation and strong magnetic field, HD 213637 belongs to the rare class of Ap stars with resolved Zeeman split spectral lines (Mathys et al. 1997) and, in fact, appears to have the second strongest mean field modulus among roAp stars. The surface field of $5.5 \mathrm{kG}$ strongly affects profiles and strengths of spectral features, so that the field cannot be neglected in abundance analysis. A single unpolarized spectrum of HD 213637 does not allow to infer detailed geometry of the magnetic field. Therefore, abundance analysis presented in Sect. 5 was based on the spectrum synthesis with the SYNTHMAG code (Piskunov 1999), which explicitly takes into account effects of polarized radiative transfer, but assumes simplified magnetic geometry, rotationally symmetric with respect to the line-of-sight. Gelbmann et al. (2000) showed that such an approach is adequate for abundance analyses of slowly rotating, strongly magnetic Ap stars.

Several Fe I spectral lines with low magnetic sensitivity were utilized to derive projected rotational velocity of HD 213637. Figure 4 shows the fit to two such lines, 


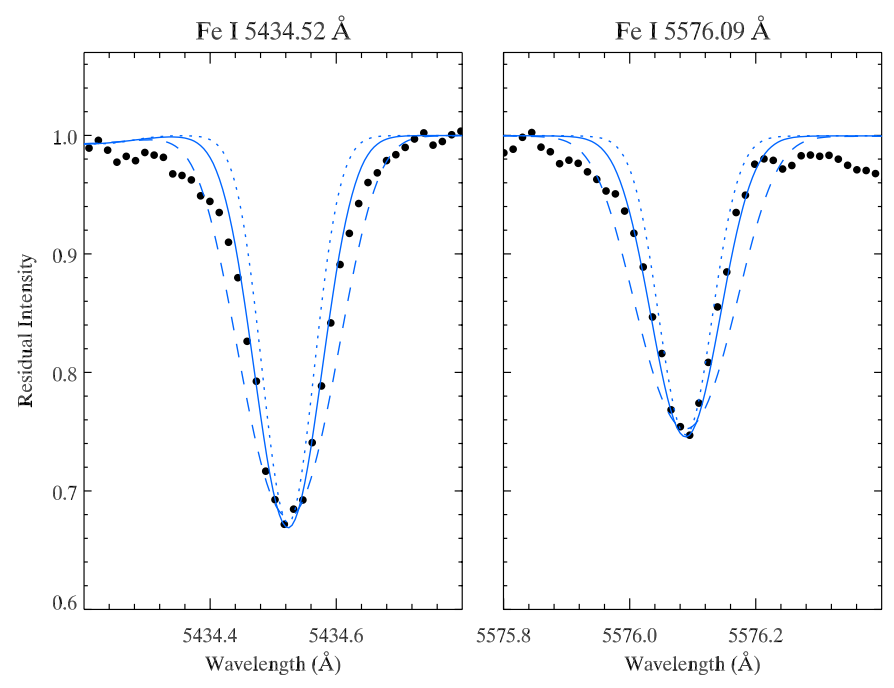

Fig. 4. Comparison between the observations (dots) and the synthetic spectra of two iron lines with low magnetic sensitivity: Fe I 5434.52 and $5576.09 \AA$ (both lines have effective Landé factors $z=-0.01$ ). The solid line corresponds to the synthetic spectra computed with $v_{\mathrm{e}} \sin i=3.5 \mathrm{~km} \mathrm{~s}^{-1}$, while the dotted and dashed lines illustrate the fit with $v_{\mathrm{e}} \sin i=0$ and $6 \mathrm{~km} \mathrm{~s}^{-1}$ respectively.

Fe I 5434.52 and $5576.09 \AA$, suggesting $v_{\mathrm{e}} \sin i=3.5 \pm$ $0.5 \mathrm{~km} \mathrm{~s}^{-1}$. With this value of rotational Doppler broadening the period of stellar rotation should be no longer than about 25 days, making HD 213637 an excellent candidate for the monitoring of the variations of longitudinal field, field modulus and other magnetic observables through the rotation cycle.

In addition to the magnetic and rotational broadening, many strong lines in the spectrum of HD 213637 have unusually wide wings. For some elements $(\mathrm{Ca}, \mathrm{Fe})$ this line shape may be produced by the vertical abundance profile with a region of low abundance overlaying part of the atmosphere with strong overabundance of metals. This picture cannot explain extended wings of doubly ionized rare-earth lines, which are probably formed in a cloud of heavy elements located very high in the stellar atmosphere (Ryabchikova et al. 2001). Additional broadening of some Pr III and Nd III lines indicate macroturbulent velocities in the range of $5-8 \mathrm{~km} \mathrm{~s}^{-1}$ (see Fig. 5) and may be related to the dramatic increase of the amplitude of non-radial oscillations towards the upper layers of roAp atmospheres (Kochukhov \& Ryabchikova 2001) or reflect a strong depth dependence of the convective motions in early F-type stars (Landstreet 1998).

\section{Element abundances}

Abundance analysis of HD 213637 was made by matching the observed line profiles and synthetic spectra. Calculations of theoretical spectra were performed with the SYNTHMAG code and using atmospheric parameters, projected rotational velocity and magnetic field strength determined in Sects. 3 and 4. In addition, in all calculations zero microturbulent velocity was assumed. In situations when a standard set of broadening parameters $\left(v_{\mathrm{e}} \sin i\right.$ and $\left.B_{\mathrm{s}}\right)$ gave poor description of the observed spectral lines, equivalent widths rather than line profile shapes were fitted.

The Vienna Atomic Line Database (VALD, Kupka et al. 1999; Ryabchikova et al. 1999) was used as a primary source of oscillator strengths and other spectral line parameters. For the rare-earth elements VALD line lists were complemented with the information from other sources as explained below.

The summary of the element abundances of HD 213637 is presented in Table 1. For each ion I give an average abundance, its formal error, derived from the scatter of individual abundances, and number of lines measured. For comparison abundances of another cool roAp star HD 122970 (Ryabchikova et al. 2000) as well as recent compilation of the solar photospheric abundances (Grevesse et al. 1996) are listed.

\subsection{Light elements}

The lithium abundance was derived from the resonance doublet at $\lambda 6708 \AA$. This line is badly blended in the spectrum of HD 213637 and large overabundance of Li listed in Table 1 is likely to be an upper limit of the actual abundance. Nevertheless, it is clear that HD 213637 belongs to the group of cool Ap stars showing significant absorption at the position where Li I doublet is expected (Polosukhina et al. 1999).

$\mathrm{C}, \mathrm{Al}, \mathrm{Si}$, and $\mathrm{S}$ are all somewhat underabundant relative to the solar chemical composition, which is typical of roAp stars.

The abundance of sodium, derived from the weak neutral lines ( $\lambda 5682$ and $5688 \AA$ ), suggests deficiency of roughly 0.7 dex. At the same time, resonance $\mathrm{Na} \mathrm{D}$ lines give larger underabundance of 1.3 dex. Very similar picture emerges from the analysis of the weak and strong lines of $\mathrm{Mg}$ I. The well-known triplet of this element ( $\lambda$ 5167, 5172, and $5183 \AA$ ) gives an order of magnitude lower abundance than derived from the medium-strength MgI line at $\lambda$ 5711.1 $\AA$. This discrepancy between abundances derived from the weak and strong lines of $\mathrm{NaI}$ and $\mathrm{Mg}$ I cannot be attributed to uncertain oscillator strengths or non-LTE effects (according to Mashonkina et al. (1993, 1996) NLTE corrections for these ions are expected to be below $0.1-0.15$ dex for main sequence stars). The $\mathrm{Na}$ and $\mathrm{Mg}$ anomaly may be attributed to an inhomogeneous vertical distribution of these elements. A vertical stratification profile, in which concentration of $\mathrm{Na}$ and $\mathrm{Mg}$ rapidly increases with depth, can qualitatively account for the observed line strengths and shapes of the $\mathrm{Na}$ I and $\mathrm{Mg}$ I lines. It is worth mentioning that other roAp stars with weak surface magnetic field do not show $\mathrm{Na}$ and $\mathrm{Mg}$ anomaly, as abundances obtained from the weak and resonance lines agree well and vertical abundance stratification of these elements is insignificant. At the same time, abundance analysis of the very peculiar roAp star HD 166473 with a $8.6 \mathrm{kG}$ magnetic field (Gelbmann et al. 2000) revealed exactly the same $\mathrm{Na}$ and $\mathrm{Mg}$ anomaly that exists in HD 213637. Thus, the present study lends strong support to the hypothesis of Ryabchikova et al. (2000) that prominent vertical stratification of $\mathrm{Na}$ and $\mathrm{Mg}$ is closely related to the presence of strong magnetic fields.

Calcium provides another example of a significant abundance stratification in the atmosphere of HD 213637. On 


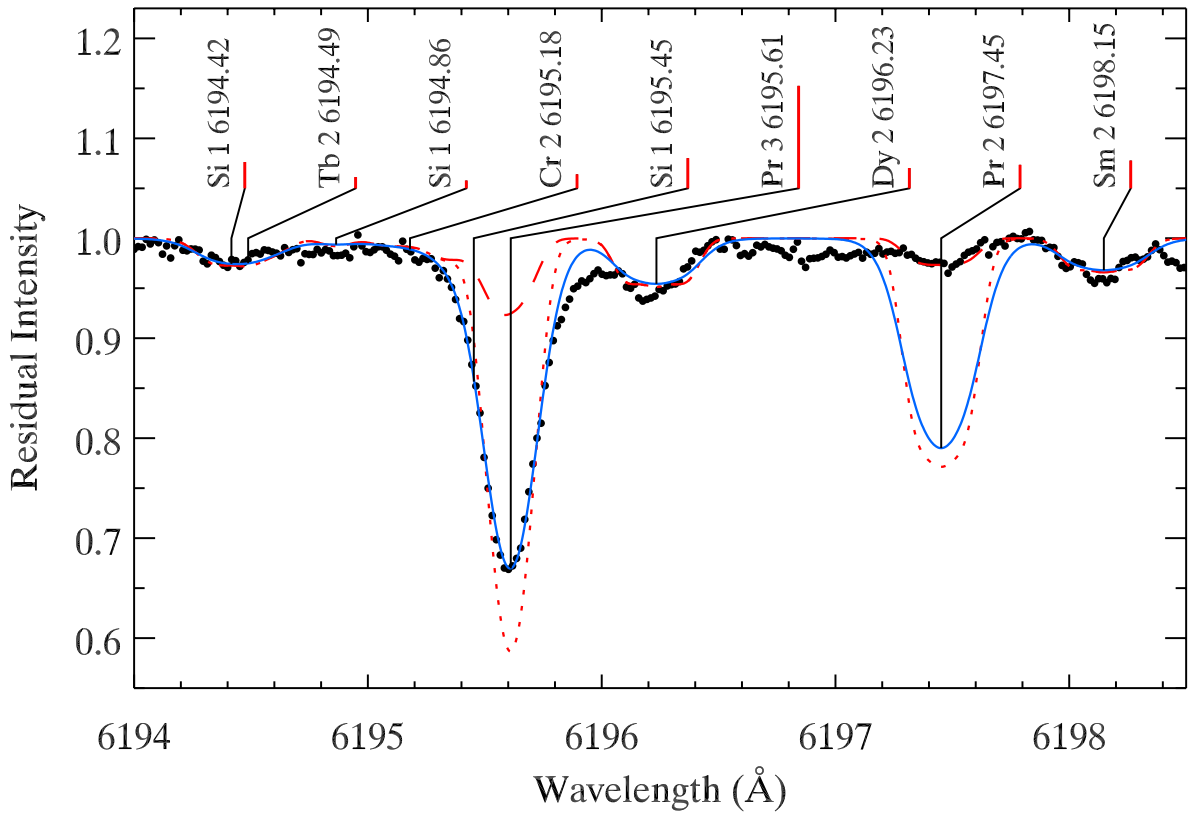

Fig. 5. Observations (dots) are compared with the spectrum synthesis in the region of the strong Pr III 6195.61 ̊ spectral line. The solid and dotted lines show calculations with $\log \left(\operatorname{Pr} / N_{\text {tot }}\right)=-7.9$, required to match equivalent width of the Pr III line, $v_{\mathrm{e}} \sin i=3.5 \mathrm{~km} \mathrm{~s}^{-1}, v_{\text {macro }}=0 \mathrm{~km} \mathrm{~s}^{-1}$ (dotted curve) and $v_{\text {macro }}=7.5 \mathrm{~km} \mathrm{~s}^{-1}$ ( (solid curve). The dashed line illustrates calculations with $v_{\mathrm{e}} \sin i=3.5 \mathrm{~km} \mathrm{~s}^{-1}$, no macroturbulent broadening and lower $\operatorname{Pr}$ abundance, $\log \left(\operatorname{Pr} / N_{\text {tot }}\right)=-9.8$, necessary to fit singly ionized lines, such as Pr II $6197.45 \AA$. average, the neutral lines of $\mathrm{Ca}$ indicate deficiency of about 0.5 dex relative to the solar photospheric chemical composition. Thus, HD 213637 appears to be more deficient in $\mathrm{Ca}$ than hotter roAp stars and is comparable in this respect to Przybylski's star, which exhibits even stronger calcium underabundance (Cowley et al. 2000). When analysed separately, the weak and strong lines of $\mathrm{Ca}$ I give abundance different by 0.4 dex. In addition, many strong $\mathrm{Ca}$ I lines show extended wings, which cannot be fitted with the adopted atmospheric parameters and an homogeneous calcium abundance. Anomalous strengths and shapes of $\mathrm{Ca}$ I lines point to a vertical stratification profile similar to that of $\mathrm{Na}$ and $\mathrm{Mg}$ : $\mathrm{Ca}$ abundance increases strongly towards the deeper atmospheric layers. Babel (1994) and Ryabchikova et al. (2002) demonstrated that this vertical distribution of $\mathrm{Ca}$ is characteristic of the atmospheres of cool Ap stars.

\subsection{Iron-peak elements}

Abundances of all iron-peak elements in the atmosphere of HD 213637 are either close to solar (Ti, V, Cr, Mn, Co) or significantly below solar concentration ( $\mathrm{Fe}, \mathrm{Ni})$. The iron and nickel deficiency in HD 213637 is similar to the abundances of these elements in the two other cool roAp stars: HD 101065 (Cowley et al. 2000) and HD 217522 (Gelbmann 1998). Thus, it seems rather well-established that iron underabundance is a common feature of the chemical composition of the atmospheres of all roAp stars with $T_{\text {eff }}$ substantially below $7000 \mathrm{~K}$.

Chromium is not dramatically enhanced in the atmosphere of HD 213637 and may be even underabundant by $\approx 0.2 \mathrm{dex}$ relative to the solar chemical composition. Therefore, the original spectral classification of HD 213637 as a Cr-peculiar star (Houk \& Smith-Moore 1988) appears to be unjustified.

The close to solar abundance of Co is a somewhat surprising property of HD 213637. In all previous abundance analyses of roAp stars cobalt was found to be overabundant by an order of magnitude or more (typically $\left.\log \left(\mathrm{Co} / N_{\text {tot }}\right)>-6.0\right)$ and it was suggested that the prominent overabundance of this element is characteristic of all roAp stars (e.g. Gelbmann et al. 2000). Results of the present abundance analysis of HD 213637 do not support this point of view.

The Fe I lines in the spectrum of HD 213637 display numerous signatures of an inhomogeneous vertical abundance distribution of iron, similar to the stratification profiles of $\mathrm{Na}$, $\mathrm{Mg}$ and $\mathrm{Ca}$ discussed in Sect. 5.1. In particular, the average abundance of Fe I, derived from the weak lines, is higher by almost 0.4 dex in comparison with the iron concentration inferred from the equivalent widths of the strong lines. In addition, many strong low-excitation lines exhibit extended wings which are impossible to fit simultaneously with the shallow cores under the assumption of an homogeneous vertical abundance distribution. These numerous pieces of evidence point to the increase of iron abundance towards the deeper atmospheric layers, very similar to the iron vertical stratification discovered by Ryabchikova et al. (2002) in the atmosphere of $\gamma$ Equ.

\section{3. $\mathrm{Sr}, \mathrm{Y}, \mathrm{Zr}$, and $\mathrm{Ba}$}

Sr, Y, and Zr are all overabundant in HD 213637 by $0.8-1.1$ dex with $\mathrm{Sr}$ being the most anomalous element in the group. This abundance pattern is typical of roAp stars. In fact, from the comparison of chemical composition of HD 213637 and HD 122970 (Table 1) one can see that abundances of Sr, Y, and $\mathrm{Zr}$ in the two stars are identical within the error bars.

Abundance of $\mathrm{Ba}$ is close to solar in HD 213637. In other roAp stars this element changes from an overabundance of about 1.0 dex (HD 122970) to a 0.4 dex deficiency ( $\alpha$ Cir, HD 166473) relative to the solar chemical composition. The average abundance of Ba in HD 213637 was determined from a sample of four Ba II lines with similar strengths and excitation potentials. This makes it difficult to assess possible vertical abundance stratification of this element. Nevertheless, there 
Table 1. Abundances of the roAp star HD 213637 based on $n$ lines. Formal error estimates in units of the last digit are given in parentheses. For comparison solar photospheric chemical composition (Grevesse et al. 1996) and element abundances of another cool roAp star HD 122970 (Ryabchikova et al. 2000) are listed.

\begin{tabular}{|c|c|c|c|c|c|c|c|c|c|}
\hline \multirow[t]{2}{*}{ Ion } & \multicolumn{2}{|c|}{ HD 213637} & \multirow{2}{*}{$\begin{array}{l}\text { HD } 122970 \\
\log \left(N / N_{\text {tot }}\right)\end{array}$} & \multirow{2}{*}{$\begin{array}{c}\odot \\
\log \left(N / N_{\text {tot }}\right)\end{array}$} & \multirow[t]{2}{*}{ Ion } & \multicolumn{2}{|c|}{ HD 213637} & \multirow{2}{*}{$\begin{array}{l}\text { HD } 122970 \\
\log \left(N / N_{\text {tot }}\right)\end{array}$} & \multirow{2}{*}{$\begin{array}{c}\odot \\
\log \left(N / N_{\text {tot }}\right)\end{array}$} \\
\hline & $\log \left(N / N_{\text {tot }}\right)$ & $n$ & & & & $\log \left(N / N_{\text {tot }}\right)$ & $n$ & & \\
\hline Li I & -9.25 & 1 & & -10.88 & $\mathrm{Cu} \mathrm{I}$ & $-8.17(23)$ & 3 & -8.13 & -7.83 \\
\hline C I & $-3.77(08)$ & 3 & -3.51 & -3.49 & $\mathrm{Zn} I$ & -8.00 & 1 & -7.80 & -7.44 \\
\hline \multirow[t]{2}{*}{$\mathrm{NaI}$} & $-6.38(04)^{\dagger}$ & 2 & -5.87 & -5.71 & SrI & $-7.80(10)$ & 3 & -7.70 & -9.07 \\
\hline & $-7.00(10)^{\ddagger}$ & 2 & & -5.71 & Y II & $-9.03(19)$ & 10 & -9.09 & -9.80 \\
\hline \multirow[t]{2}{*}{ Mg I } & $-4.80^{\dagger}$ & 1 & -4.50 & -4.46 & Zr II & $-8.53(06)$ & 3 & -8.48 & -9.44 \\
\hline & $-5.80^{\ddagger}$ & 1 & & -4.46 & Mo I & $-8.53(17)$ & 2 & & -10.12 \\
\hline $\mathrm{Al}$ I & -5.80 & 1 & -5.73 & -5.57 & Ba II & $-9.81(09)$ & 4 & -8.99 & -9.91 \\
\hline Si I & $-4.85(12)$ & 12 & -4.45 & -4.49 & La II & $-9.41(14)$ & 25 & -9.34 & -10.87 \\
\hline Si II & $-4.84(23)$ & 2 & -4.24 & -4.49 & Ce II & $-9.02(16)$ & 18 & -8.82 & -10.46 \\
\hline S I & $-5.11(19)$ & 3 & -4.83 & -4.71 & Pr II & $-9.91(11)$ & 14 & -10.04 & -11.33 \\
\hline \multirow[t]{3}{*}{$\mathrm{Ca} \mathrm{I}$} & $-6.14(29)$ & 16 & -5.48 & -5.68 & Pr III & $-8.43(15)$ & 8 & -8.63 & -11.33 \\
\hline & $-5.98(20)^{\dagger}$ & 10 & & -5.68 & Nd II & $-9.05(18)$ & 56 & -9.23 & -10.54 \\
\hline & $-6.41(20)^{\ddagger}$ & 6 & & -5.68 & Nd III & $-7.53(12)$ & 8 & -8.03 & -10.54 \\
\hline Ca II & -5.60 & 1 & -5.43 & -5.68 & Sm II & $-9.15(14)$ & 25 & -9.33 & -11.03 \\
\hline Sc II & -9.70 & 1 & -8.66 & -8.87 & Eu II & $-9.81(32)$ & 3 & -9.66 & -11.53 \\
\hline Ti I & $-7.20(20)$ & 4 & -6.91 & -7.02 & Gd II & $-8.72(16)$ & 22 & -8.67 & -10.92 \\
\hline Ti II & $-7.62(09)$ & 8 & -6.88 & -7.02 & Tb II & $-9.90(10)$ & 3 & -10.00 & -12.14 \\
\hline V I & -7.75 & 1 & -7.84 & -8.04 & Tb III & $-7.86(21)$ & 13 & -8.31 & -12.14 \\
\hline V II & -7.70 & 1 & -7.73 & -8.04 & Dy II & $-9.03(21)$ & 8 & -9.07 & -10.90 \\
\hline $\mathrm{CrI}$ & $-6.62(17)$ & 11 & -5.99 & -6.37 & Dy III & -7.40 & 1 & & -10.90 \\
\hline Cr II & $-6.59(17)$ & 12 & -6.06 & -6.37 & Er II & $-9.38(24)$ & 6 & -9.63 & -11.11 \\
\hline Mn I & $-6.79(18)$ & 3 & -6.40 & -6.65 & Er III & $-7.27(05)$ & 2 & -7.60 & -11.11 \\
\hline Mn II & -6.18 & 1 & -6.31 & -6.65 & Tm II & $-9.24(29)$ & 8 & -9.04 & -12.04 \\
\hline \multirow[t]{3}{*}{ Fe I } & $-5.37(28)$ & 62 & -4.48 & -4.54 & Yb II & $-10.50(20)$ & 3 & -9.83 & -11.02 \\
\hline & $-5.20(20)^{\dagger}$ & 33 & & -4.54 & Lu II & $-9.84(06)$ & 4 & -9.75 & -11.28 \\
\hline & $-5.57(22)^{\ddagger}$ & 29 & & -4.54 & & & & & \\
\hline Fe II & $-5.37(09)$ & 9 & -4.46 & -4.54 & $T_{\text {eff }}(\mathrm{K})$ & \multicolumn{2}{|c|}{$6400 \pm 100$} & $6930 \pm 100$ & 5777 \\
\hline Co I & $-6.80(07)$ & 4 & -6.06 & -7.12 & $\log g$ & \multicolumn{2}{|c|}{$3.60 \pm 0.2$} & $4.11 \pm 0.1$ & 4.44 \\
\hline $\mathrm{Ni} I$ & $-6.82(13)$ & 4 & -5.89 & -5.79 & $B_{\mathrm{s}}(\mathrm{kG})$ & \multicolumn{2}{|c|}{$5.5 \pm 0.1$} & 2.3 & \\
\hline
\end{tabular}

† Abundance derived from weak lines.

$\$$ Abundance derived from strong lines.

is marginal evidence for an increase of $\mathrm{Ba}$ abundance in the deeper atmospheric layers, as the strong Ba II lines have wider wings than predicted by the spectrum synthesis with an homogeneous abundance (see Fig. 3 for the example of such a discrepancy between the observed and computed profiles of the Ba II $6141.7 \AA$ line).

\subsection{Rare-earth elements}

In the analysis of REE lines in the spectrum of HD 213637 the VALD line lists were supplemented by the information from the DREAM database ${ }^{1}$ (Biémont et al. 1999). In addition, for the study of doubly ionized Pr III and Nd III spectral lines the calculations of Bord (2000 and private communication) were used

\footnotetext{
${ }^{1}$ http://www.umh.ac.be/ ${ }^{\sim}$ astro/dream.shtml
}

with the astrophysical oscillator strengths taken from the study of Dolk et al. (2002). Theoretical Landé factors of the energy levels of Er III were kindly made available by P. Quinet (private communication).

In general, abundances of all REE (based on first ions) in the atmosphere of HD 213637 agree well with the rare-earth abundance pattern found for other roAp stars (e.g. Ryabchikova et al. 2000). Also, similar to the results of the previous studies, analysis of the spectrum of HD 213637 revealed striking discrepancy between abundances obtained from the first and second ions of several rare-earth elements. In particular, the Pr III, $\mathrm{Nd}$ III, and Dy III lines indicate $\approx 1.5$ dex higher abundance than determined from lines of the first ions, while for Tb III and Er III this difference reaches 2.0 dex!

Ryabchikova et al. (2001) demonstrated that for Pr and Nd the REE anomaly can not be explained by systematic errors of 
the oscillator strengths and is observed exclusively in the pulsating Ap stars. In the study of Ryabchikova et al. (2002) it was proposed that anomalously strong doubly ionized REE lines observed in the spectrum of the roAp star $\gamma$ Equ can be explained by the presence of an overabundant layer of rareearth elements in the upper atmosphere. This vertical stratification scenario has indeed provided satisfactory agreement between observed and computed strengths of the $\mathrm{Pr}$ and $\mathrm{Nd}$ lines in $\gamma$ Equ. Furthermore, pulsational radial velocity studies of roAp stars (Kochukhov \& Ryabchikova 2001; Balona 2002) demonstrated that doubly ionized REE lines dominate pulsational spectroscopic variability and show pulsational amplitudes which by orders of magnitude exceed those observed for other metal lines, but at the same time comparable to the pulsations seen in the $\mathrm{H} \alpha$ core. This confirms that the lines of second REE ions form in the upper atmospheric layers where non-radial pulsations attain high amplitudes.

Thus it seems plausible to suggest that the REE anomaly observed in HD 213637 can also be attributed to a vertical stratification of the abundances of $\mathrm{Pr}, \mathrm{Nd}, \mathrm{Tb}, \mathrm{Dy}$, and Er. All these elements are expected to be concentrated in the upper atmosphere, above optical depth $\log \tau_{5000} \approx-8$.

\section{Discussion}

The temperature derived for HD 213637 in this study is one of the lowest $T_{\text {eff }}$ determinations in a modern spectrum synthesis analysis of a magnetic chemically peculiar star. In fact, only Przybylski's star (HD 101065) was suggested to be even cooler $\left(T_{\text {eff }}=6300 \mathrm{~K}\right.$, Kochukhov et al. 2002). However, analysis of this extreme object is very difficult and uncertain due to the prominent influence of anomalous line blanketing on the model atmosphere structure (Piskunov \& Kupka 2001). In contrast, HD 213637 has a relatively moderate enhancement of the heavy elements and a straightforward fit to the Balmer lines using standard model atmospheres is probably sufficient to determine $T_{\text {eff }}$ with an accuracy of $\approx 100 \mathrm{~K}$. The temperature of HD 213637 suggests that the cool border of the region in the HR diagram occupied by magnetic peculiar stars lies at about $500 \mathrm{~K}$ lower temperature than commonly assumed. Furthermore, clear evidence for the presence of strong vertical abundance gradients in the stellar atmosphere indicates that magnetic field fully inhibits convective and turbulent mixing in the outer stellar envelope. Elucidating evolutionary stage of HD 213637 is crucial in understanding whether its characteristics (low temperature, strong magnetic field, and strong abundance gradients) belong to a low-mass star which is still on the main sequence or to a more massive, typical cool Ap star already evolving towards the giant phase.

Abundance analyses of the cool roAp stars revealed many new intriguing characteristics, which challenge some of the properties, commonly attributed to magnetic Ap stars. For example, it was widely believed that chemical diffusion always produces overabundance of iron-peak elements in the magnetic chemically peculiar stars. However, recent self-consistent diffusion calculations by F. LeBlanc and co-workers (e.g. Ryabchikova et al. 2003) illustrate that in the cool Ap stars high concentration of iron-peak elements occurs only in deep

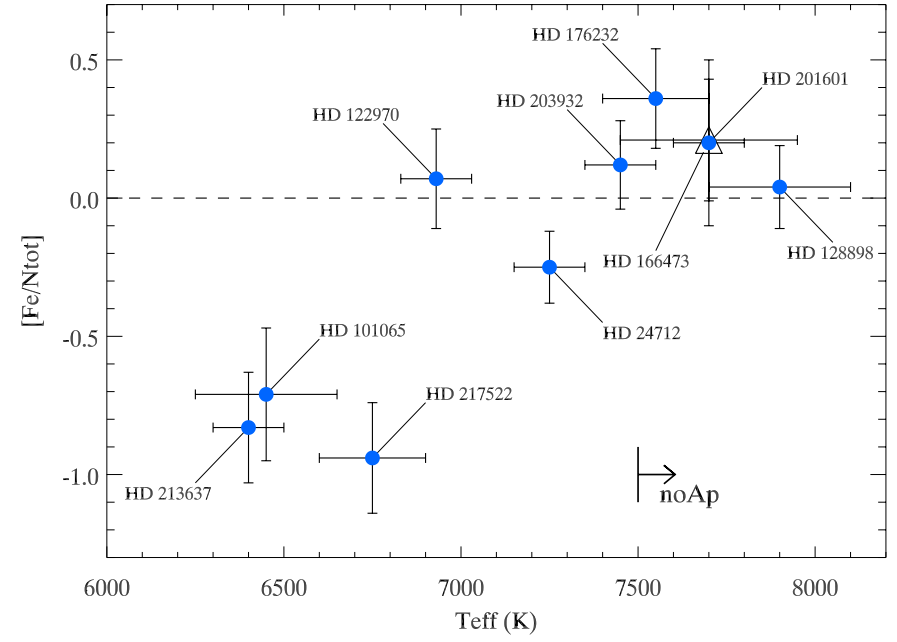

Fig. 6. Effective temperatures and iron abundances (relative to the sun) of the roAp stars. The vertical bar in the lower part of the figure shows approximate temperature of the coolest known non-pulsating Ap stars. A different symbol has been chosen to represent HD 166473 in order to avoid an overlap with HD 201601.

atmospheric layers, but it is plausible to expect sharp decrease of Fe abundance above optical depth $\log \tau_{5000} \approx-1$. As a result, depth-averaged iron abundance is only marginally enhanced in Ap stars with $T_{\text {eff }} \leq 8000$. This is corroborated by observations, as in hotter roAp stars iron abundance appears close to solar, while $\mathrm{Fe}$ is underabundant by up to one order of magnitude in all Ap stars with effective temperatures substantially below $7000 \mathrm{~K}$. HD 213637 fits well in this picture and confirms the existence of a general correlation between metallicity and $T_{\text {eff }}$ (Gelbmann 1998). This is illustrated in Fig. 6, which combines information on effective temperatures and iron abundances of all roAp stars analysed until now (Gelbmann 1998; Ryabchikova et al. 2000 and references therein; this study). Clear correlation between $T_{\text {eff }}$ and $\log \left(\mathrm{Fe} / N_{\text {tot }}\right)$ may be related to a systematic change of the optical depth of the $\mathrm{Fe}$ abundance jump with effective temperature.

Another interesting observation is the absence of cool nonpulsating Ap (noAp) stars: apparently there are no main sequence noAp stars with $T_{\text {eff }} \lessgtr 7500 \mathrm{~K}$. It would be interesting to clarify whether this effect is produced by some unrecognized observational bias together with the scarcity of modern spectroscopic studies of cool noAp stars, or the onset of pulsations is physically related to low $T_{\text {eff }}$.

Since HD 213637 lies at the border of the class of pulsating Ap stars, it is interesting to compare its atmospheric parameters with the theoretical predictions for the red edge of the roAp instability strip. The mechanism exciting pulsations in roAp stars remains highly uncertain and several competing hypotheses were put forward to explain its origin. In this situation atmospheric analysis of the extreme members of the roAp group offers unique possibility to select theoretical models consistent with observations. First, it should be noted that, although in the past roAp stars were commonly believed to share the same instability strip as $\delta$ Scuti stars, recent spectroscopic studies revealed many roAp stars to lie beyond the cooler edge of the empirically determined $\delta$ Scuti instability strip (which, according 
to Rodriguez et al. (1994), is located at about $T_{\text {eff }}=7100 \mathrm{~K}$ for the main sequence stars). HD 213637 provides the most clear example of this discrepancy. Thus it seems unlikely that magnetic pulsating stars are excited by the same He II ionization instability mechanism that works in $\delta$ Scuti pulsators. A similar conclusion was reached by Matthews et al. (1999) from the analysis of asteroseismic and Hipparcos luminosities of roAp stars. Spectroscopic temperatures of some magnetic peculiar stars also tend to rule out the Si IV ionization mechanism (Matthews 1988), which predicts a boundary of the roAp instability strip only about $300 \mathrm{~K}$ cooler that the He II strip.

Gautschy et al. (1998) showed that the $\kappa$ mechanism of $\mathrm{H} / \mathrm{He}$ ionization successfully drives short-period oscillation modes in roAp stars, provided that stellar models have a temperature inversion in the inner atmosphere. The location and form of this temperature anomaly is qualitatively similar to the hot atmospheric layer found by Kochukhov et al. (2002) from the semi-empirical analysis of the hydrogen lines in some cool Ap stars. The model of Gautschy et al. well accounts for the excitation of acoustic $p$-modes in cooler roAp stars, but predicts the blue edge of the roAp instability strip at about $T_{\text {eff }}=7250 \mathrm{~K}$. This is too cool to account for the majority of roAp stars with effective temperatures in the range between roughly 7500 and $8000 \mathrm{~K}$.

The latest attempt to understand the roAp excitation mechanism was made by Balmforth et al. (2001). They suggested that near the magnetic poles of roAp stars convection is totally suppressed by the magnetic forces and stellar atmosphere is chemically differentiated, whereas near the equator of dipolar magnetic field convective motions are not inhibited and atmosphere is chemically homogeneous. The excitation of non-radial pulsation modes occurs in this composite model due to the $\kappa$ mechanism operating in the hydrogen ionization zone in the polar regions. Cunha (2002) carried out detailed investigation of the theoretical instability strip predicted by the model of Balmforth et al. (2001). She found that the coolest roAp stars, in which high-order acoustic oscillations are excited, correspond to the most evolved stellar models with effective temperatures no less than $\approx 7150 \mathrm{~K}$. Thus, predictions of the excitation mechanism of Balmforth et al. (2001) are clearly contradicted by the observations of non-radial pulsations in HD 213637 and other roAp stars with $T_{\text {eff }} \leq 7000 \mathrm{~K}$.

Acknowledgements. I thank Eric Stempels for obtaining the spectrum of HD 213637. This paper benefited a lot from many stimulating discussions with Dr. Tanya Ryabchikova and her helpful advice on atomic data. I also sincerely thank the referee, Dr. M. Landi, whose constructive remarks contributed to the improvement of this paper.

\section{References}

Babel, J. 1994, A\&A, 283, 189

Balmforth, N. J., Cunha, M. S., Dolez, N., Gough, D. O., \& Vauclair, S. 2001, MNRAS, 323, 362

Balona, L. A. 2002, MNRAS, 337, 1059
Barklem, P. S., Piskunov, N., \& O’Mara, B. J. 2000, A\&A, 363, 1091

Biémont, E., Palmeri, P., \& Quinet, P. 1999, Ap\&SS, 269, 635

Bord, D. J. 2000, A\&AS, 144, 517

Cunha, M. S. 2002, MNRAS, 333, 47

Cowley, C. R., Ryabchikova, T., Kupka, F., et al. 2000, MNRAS, 317, 299

Dolk, L., Wahlgren, G. M., Lundberg, H., et al. 2002, A\&A, 385, 111

Gautschy, A., Saio, H., \& Harzenmoser, H. 1998, MNRAS, 301, 31

Gelbmann, M. J. 1998, Contrib. Astron. Obs. Skalnaté Pleso, 27, 280

Gelbmann, M., Ryabchikova, T., Weiss, W. W., et al. 2000, A\&A, 356, 200

Grevesse, N., Noels, A., \& Sauval, A. J. 1996, in Cosmic Abundances, ed. S. S. Holt, \& G. Sonneborn, ASP Conf. Ser., 99, 117

Houk, N., \& Smith-Moore, M. 1988, Michigan Spectral Catalogue of Two-Dimensional Spectral Types for the HD Stars - Vol. 4, Department of Astronomy, University of Michigan, Ann Arbor

Kochukhov, O., \& Ryabchikova, T. 2001, A\&A, 374, 615

Kochukhov, O., Bagnulo, S., \& Barklem, P. S. 2002, ApJ, 578, 75

Kupka, F., Piskunov, N., Ryabchikova, T. A., Stempels, H. C., \& Weiss, W. W. 1999, A\&AS, 138, 119

Kurtz, D. W., \& Wegner, G. 1979, ApJ, 232, 510

Kurtz, D. W., \& Martinez, P. 2000, Baltic Astron., 9, 253

Kurucz, R. L. 1993, ATLAS9 Stellar Atmosphere Programs, CD-ROM No. 13 (Smithsonian Ap. Obs.)

Landstreet, J. D. 1998, A\&A, 338, 1041

Martinez, P., Meintjes, P., Ratclif, S. J., \& Engelbrecht, C. 1998, A\&A, 334, 606

Mashonkina, L. I., Sakhibullin, N. A., \& Shimanskij, V. V. 1993, Astron. Rep., 37, 192

Mashonkina, L. I., Shimanskaya, N. N., \& Sakhibullin, N. A. 1996, Astron. Rep., 40, 187

Mathys, G., Hubrig, S., Landstreet, J. D., Lanz, T., \& Manfroid, J. 1997, A\&AS, 123, 353

Matthews, J. M. 1988, MNRAS, 235, 7P

Matthews, J. M., Kurtz, D. W., \& Martinez, P. 1999, ApJ, 511, 422

Piskunov, N. 1992, in Stellar Magnetism, ed. Yu. V. Glagolevskij, \& I. I. Romanyuk (St. Petersburg: Nauka), 92

Piskunov, N. E. 1999, in 2nd International Workshop on Solar Polarization, ed. K. Nagendra, \& J. Stenflo (Kluwer Acad. Publ.), ASSL, 243, 515

Piskunov, N., \& Kupka, F. 2001, ApJ, 547, 1040

Polosukhina, N., Kurtz, D., Hack, M., et al. 1999, A\&A, 351, 283

Rodriguez, E., Lopez de Coca, P., Rolland, A., Garrido, R., \& Costa, V. 1994, A\&AS, 106, 21

Rogers, N. Y. 1995, Comm. Asteroseismol., 78

Ryabchikova, T. A., Piskunov, N. E., Stempels, H. C., Kupka, F., \& Weiss, W. W. 1999, Phys. Scr., T83, 162

Ryabchikova, T. A., Savanov, I. S., Hatzes, A. P., Weiss, W. W., \& Handler, G. 2000, A\&A, 357, 981

Ryabchikova, T. A., Savanov, I. S., Malanushenko, V. P., \& Kudryavtsev, D. O. 2001, Astron. Rep., 45, 382

Ryabchikova, T., Piskunov, N., Kochukhov, O., et al. 2002, A\&A, 384, 545

Ryabchikova, T. A., Wade, G. A., \& LeBlanc, F. 2003, in IAU Symp. 210, Modelling of Stellar Atmospheres, ed. N. Piskunov, W. W. Weiss, \& D. F. Gray, ASP Conf. Ser., in press

Schaller, G., Schaerer, D., Meynet, G., \& Maeder, A. 1992, A\&AS, 96, 269 